\title{
Unified Analysis of Thermodynamic and Rheological Properties of High Polymer Solutions. I. Binary Systems
}

\author{
TOMÁS S. GRIGERA,* ISABEL M. IRURZUN, M. SUSANA CORTIZO, RUBÉN V. FIGINI, ${ }^{+}$ \\ MARIANNE MARX-FIGINI \\ Instituto de Investigaciones Fisicoqumícas Teóricas y Aplicadas (INIFTA), CONICET, CIC y Universidad Nacional de La \\ Plata, C.C. 16, suc. 4, 1900 La Plata, Argentina
}

Received 29 January 2001; revised 21 September 2001; accepted 9 November 2001

\begin{abstract}
We present a comprehensive experimental study of thermodynamic and rheological properties of semidilute polymer solutions in good solvent. Osmotic pressure and viscosity measurements have been done in several polymer-solvent systems at different temperatures. A renormalization group technique was applied to analyze the data using de Gennes's blobs model to connect dynamic and conformational quantities. The behavior of polymer systems in the whole range from dilute to semidilute solutions can be satisfactorily described using only a few nonuniversal quantities experimentally determined. An adequate agreement between experiments and theory was found, showing universal behavior with a system-dependent constant $\beta$ that does not depend on molecular weight or concentration. (C) 2001 John Wiley \& Sons, Inc. J Polym Sci Part B: Polym Phys 40: 290-301, 2002
\end{abstract}

Keywords: heterogenous polymers; viscosity; thermodynamics

\section{INTRODUCTION}

It has been known for a long time that polymer solutions show universal behavior; macroscopic properties depend on variables such as molecular weight and concentration through general relationships that hold for a wide range of chemically different systems. ${ }^{1-3}$ The details of the microscopic interactions (system and temperature dependent) are hidden in a few nonuniversal constants. The power laws (such as Flory's equation) derived from scaling arguments are perhaps the best known manifestation of universality.

*Present address: Gruppo VIM, Dipartimento di Fisica, Universita' di Roma 'La Sapienza,' P.le A. Moro, 2, Roma I-00185, Italy

'Deceased

Correspondence to: T. S. Grigera (E-mail: tomas.grigera@ roma1.infn.it)

Journal of Polymer Science: Part B: Polymer Physics, Vol. 40, 290-301 (2002)

(C) 2001 John Wiley \& Sons, Inc.

DOI 10.1002/polb.10089
However, power laws are only valid in the limiting cases of infinitely dilute and semidilute solutions. In the crossover region universality still holds, but the scaling hypothesis is not valid, and the relations do not have a simple form. The powerful renormalization group (RG) techniques are needed to derive these relations from a microscopic model. ${ }^{4,5}$ Power laws are then obtained in the appropriate limits.

To date, reasonable understanding of dilute solutions exists, and extensive experimental information allows the prediction of their properties from the knowledge of a few nonuniversal quantities. ${ }^{6,7}$ Progress has also been made in the description of semidilute solutions, and now thermodynamic and conformational properties can be similarly accounted for. ${ }^{8-11}$ The treatment of dynamic properties, on the other hand, is more difficult because of the screening of the hydrodynamic interaction. ${ }^{12-14}$

Despite these progressions, we feel that from the experimental point of view a comprehensive 
analysis is still required. In a previous article $\mathrm{e}^{11}$ we presented an analysis of viscosity and dielectric relaxation times of cellulose trinitrate solutions on the basis of $R G$ results. Here we attempt to consider static and dynamic data under a common scheme. We report extensive experimental measurements of osmotic pressure and Newtonian viscosity of polymer solutions across the dilute-semidilute transition, comprising several chemically different systems and temperatures. Results are analyzed using the renormalization group scheme developed by Schäfer that allows the computation of quantities such as osmotic pressure and radius of gyration. To extend our study to dynamic properties, a relationship between them and conformational properties was established using scaling arguments from the de Gennes's blob model. Nonuniversal quantities were identified and calculated, showing that the overall behavior can be satisfactorily described.

Elsewhere we have applied the same scheme to the description of thermodynamic and rheological properties of ternary polymer solutions, thus achieving a unified approach to the description of all these systems. ${ }^{15}$

This article is organized as follows. First, we summarize the relevant RG results. Second, we give details on the samples and experimental measurements. Third, we discuss the results and finally our conclusions.

\section{THEORETICAL BACKGROUND}

Experimentally, it has been well established that from the dilute to the semidilute regime, macroscopic properties of polymer solutions are universal functions of the overlap parameter $c / c^{*}$, where $c^{*}$ is a critical concentration at which the polymer coils begin to overlap. ${ }^{16,17} c^{*}$ is usually defined as

$$
c^{*}=\frac{3 M}{4 \pi S_{\mathrm{G}, 0}^{3} N_{\mathrm{A}}}
$$

where $M$ is the molar mass of the solute, $N_{\mathrm{A}}$ is Avogadro's number, and $S_{\mathrm{G}, 0}$ is the radius of gyration of a single chain.

A few years ago, Schäfer developed a renormalization group approach for the description of conformational and thermodynamic universal properties of polymer solutions. ${ }^{8-10}$ This theory provides a unified treatment of temperature and concentration crossover of excluded volume inter- actions, providing a single computational scheme for all physically observable quantities. The system is described using the well-known Gaussian model with excluded volume interactions. The polymer chain is characterized by a microscopic length $l$ and a quantity $\mu,{ }^{18}$ representing the strength of the two-body interaction in the cluster expansion. The solution is defined by the number concentrations of polymer chains $c_{\mathrm{p}}$, a numberaverage chain length $N$, and a chain-length distribution $P(n)$.

In this scheme, ${ }^{9}$ all macroscopic quantities can be expressed in terms of two variables, $f$ and $w \cdot f$ is related to the excluded volume interaction, and it is normalized so that $f=0$ in the $\Theta$ state and $f$ $=1$ in the good solvent limit. Variable $w$ must be introduced in the description of the concentration crossover to consider the screening of the excluded volume. Because the interactions are screened beyond the correlation length $\xi$, the renormalization procedure must be carried out until the characteristic length $l$ essentially coincides with $\xi$ ( $\xi$ approaches $S_{\mathrm{G}}$ in the infinite dilution limit). Because $\xi$ depends on both molecular weight and concentration, this requires the introduction of $w$. Variable $w$ runs from 1 in the dilute limit to 0 in the semidilute case. Thus, the RG theory incorporates in a rigorous way the concept of blobs. ${ }^{9}$

Physical quantities are obtained as a perturbative series in $w$ and $f$. Perturbative results are generally available up to first order. Second-order calculations exist for osmotic pressure in some particular cases, but we prefer to use the firstorder expansion to compare the results of different properties.

The connection with experiment is realized by writing measurable variables $c_{1}$ and $N\left(c_{l}=c_{\mathrm{p}} \cdot N\right)$ in terms of $w$ and $f$. The procedure is called a mapping of unrenormalized (or bare) variables onto renormalized scaling field ones and needs the introduction of two nonuniversal scale functions $s_{N}$ and $s_{l}$ that absorb the microscopic details of the systems. Except for the value of these functions, the $\left(c_{l}, N\right)$ dependence is universal.

An experiment traces out a path in $w-f$ space, called a crossover diagram. ${ }^{9}$ In particular, starting from a dilute solution $(w=1)$ in the excluded volume regime $(f=1)$ and increasing the concentration, a double crossover can be observed; there is a concentration transition $(w=1 \rightarrow w=0)$ in the excluded volume regime $(f=1)$ followed by a "temperature" crossover in the semidilute regime $(w=0)$. Actually, the path is not a step function 
in $w$ - $f$ space, that is, both variables change simultaneously. However, at high molecular weights the first crossovers happens much more quickly than the second, and it is correct to speak of a double crossover to a good approximation. As a result of the molecular weights and concentration ranges covered in these experiments, the second crossover is not observed, and it is reasonable to use $f=1$. In this limit, the universal functions take the simplest form. On the one hand, $f$ drops out and we are left with expansions of all observables in terms of $w$ only. On the other hand, both nonuniversal scaling functions $s_{\mathrm{N}}$ and $s_{1}$ are combined in a single quantity $B=s_{l} s_{N}{ }^{\nu}$, where $\nu$ $=0.588$ is the excluded volume critical exponent. The mapping between $w$ and the experimental variables $\left(c \propto c_{l}, M_{\mathrm{n}} \propto N\right)$ is then obtained through the overlap parameter $s\left(\propto c / c^{*}\right)$ that for $f=1$ is written $\mathrm{as}^{9}$

$$
s=0.5(1-w) w^{1-3 v}\left(\frac{9}{8}+\frac{1}{4} \frac{w^{1 / 2}}{1+w^{1 / 2}}\right)^{3 v}
$$

in terms of $w$, and

$$
s=\beta^{3} c M_{\mathrm{n}}^{3 \nu-1}
$$

where $c$ is the polymer concentration in grams per milliliter, and $M_{\mathrm{n}}$ is the number-average molecular weight. $\beta(\beta \propto B)$ is the nonuniversal quantity and cannot be calculated from theory but must be determined by fitting experimental data to theoretical expressions. However, we note that $\beta$ should be independent of molecular weight and concentration.

Once $\beta$ is fixed, all observables become functions of $s$ parametrically through $w .^{9}$ For instance, the osmotic pressure $\Pi$ can be written as

$$
\frac{\Pi}{\Pi_{0}}=\frac{17}{16}+\frac{13(1-w)}{32 w}+\frac{(1 / 8 w-1 / 4) w^{1 / 2}}{1+w^{1 / 2}}
$$

where $\Pi_{0}$ is Van't Hoff's value $\Pi_{0}=R T c / M_{\mathrm{n}}$, where $\mathrm{R}=8.314 \mathrm{~J} /(\mathrm{mol} \cdot \mathrm{K})$, and $T$ is the absolute temperature.

The radius of gyration is given by

$$
\frac{S^{2}}{S_{0}^{2}}=1.30 w^{-1}\left[\frac{w}{\frac{9}{8}+\frac{1}{4} \frac{w^{1 / 2}}{\left(1+w^{1 / 2}\right)}}\right]^{2 v} \frac{[1+\Delta(w)]}{[1+\Delta(1)]}
$$

where $\Delta(w)$ is a function of polydispersity, and $S_{0}$ is the radius of gyration at infinite dilution. For monodisperse systems $\Delta(w)$ is given by

$$
\begin{array}{r}
\Delta(w)=0.062-0.240 w^{1 / 2}-0.008 w+0.265 w^{3 / 2} \\
-0.142 w^{2} \quad(6 \mathrm{a})
\end{array}
$$

whereas for an exponential chain-length distribution $^{19}$

$$
\begin{array}{r}
\Delta(w)=0.062+0.173 w-0.141 w^{2} \\
\quad(0 \leq w \leq 1)
\end{array}
$$

We also use these results to interpret dynamic properties. Using de Gennes's reptation theory (as has been done; ${ }^{11}$ see appendix), we can obtain the following relationship between the radius of gyration and the viscosity:

$$
\frac{\eta_{\mathrm{sp}}}{[\eta] c}=\left(\frac{S^{2}}{S_{0}^{2}}\right)^{(3 \nu-4) /(2 \nu-1)}
$$

where $\eta_{\mathrm{sp}}$ and $[\eta]$ are the specific and intrinsic viscosities, respectively. This approximate equation is obtained within the scaling theory in the semidilute regime. It has the expected dilute and semidilute limiting behavior (Zimm and de Gennes, respectively). Because there are no rigorous $\mathrm{RG}$ results for the viscosity, we use it to describe the full crossover.

Some remarks should be made regarding the use of eq 7 in the crossover. As it is well known, when the concentration increases, chains begin to overlap, and the interactions within a chain are modified by the presence of the other chains. The static consequence is the screening of the excluded volume interactions, and the concomitant dynamic feature is that the hydrodynamic interaction is also screened. The relaxation times change from a Zimm behavior to a Rouse one. At still higher overlap, chains begin to entangle and reptation dynamics (de Gennes) sets in. In eq 7 the excluded volume screening is indirectly considered through the RG treatment of the radius of gyration. This has the effect of smoothly interpolating between the Zimm and de Gennes exponents (see appendix). Although it is only a crude approximation, it roughly gives the correct behavior in the crossover, and we thus prefer to avoid other procedures as explained in the ensuing discussion. 
From eqs 2, 3, and 5-7 the dilute limit for viscosity (see appendix) is obtained as

$$
\begin{aligned}
\frac{\eta_{\mathrm{sp}}}{c}=[\eta]- & 2[\eta](3 \nu-4)(4 / 5)^{3 \nu} s+O\left(s^{2}\right) \\
& \approx[\eta]-2[\eta](3 \nu-4)(4 / 5)^{3 \nu} \beta^{3} M_{\mathrm{n}}^{3 \nu-1} c
\end{aligned}
$$

Comparing eq 8 with Schulz-Blaschke's formula (eq 11) and considering that $[\eta] \approx \eta_{\mathrm{sp}} / c($ as $c \rightarrow 0)$, the constant $k_{\mathrm{B}}$ can be written as

$$
k_{\mathrm{B}}=3.017 \frac{M_{\mathrm{n}}^{3 \nu-1}}{[\eta]} \beta^{3}
$$

Equations 4, 7, and 9 give three different ways to compute $\beta$. We have used these expressions in many chemically different systems.

\section{EXPERIMENTAL}

\section{Materials}

To explore the applicability of the theoretical framework discussed previously, we should study as many polymer-solvent systems as possible. For this study, we used poly(methyl methacrylate) (PMMA), polystyrene (PS), poly(styrene-comethyl methacrylate) (CoSM) with compositions ranging from 25 to $75 \%$, and cellulose trinitrate (CTN). For the solutions we used toluene, isophorone (3,5,5-trimethylciclohexenone), and tetrahydrofuran (analytical grade, provided by Merck, J. T. Baker, and Sintorgan) distilled before use.

Standard PS samples were obtained from Pressure Chemical Co. PMMA and CoSM were synthesized by mass radical polymerization with purified azobisisobutyronitrile (Merck) as a radical initiator. Monomers were provided by Pasa (styrene) and Fluka (methyl methacrylate). Both were washed with a $10 \%$ sodium hydroxide solution to eliminate the inhibitor, then with distilled water, finally dried with calcium chloride, and distilled before use. Polymerization was carried out under vacuum at $50{ }^{\circ} \mathrm{C}$ for PMMA and $40{ }^{\circ} \mathrm{C}$ for CoSM. Reaction times (from 3 to $72 \mathrm{~h}$ ) were chosen to obtain a conversion no greater than $10 \%$. After synthesis, the polymers were purified by multiple reprecipitation in methanol.

The cellulose trinitrate samples were prepared from native cotton as previously reported. ${ }^{20}$ With this procedure, samples with a nitrogen content of
$13.9 \pm 0.5 \%$ (corresponding to a substitution $S$ $=2,90 \pm 0,02)$ were obtained.

\section{Characterization}

\section{Copolymer Composition}

The composition of the copolymer samples was determined by elemental chemical analysis or calculated from the expression derived from the terminal model of radical copolymerization. ${ }^{21}$ For two monomers $\mathbf{1}$ and $\mathbf{2}$, the molar fraction of $\mathbf{1}$ in the polymer is given by

$$
N_{1}=\frac{r_{1} n_{1}^{2}+n_{1} n_{2}}{r_{1} n_{1}^{2}+2 n_{1} n_{2}+r_{2} n_{2}^{2}}
$$

where $n_{1}$ and $n_{2}$ are the molar fractions of the monomers in the reactive mixture, and $r_{\mathbf{1}}$ and $r_{\mathbf{2}}$ are the reactivity coefficients. In our case, $\mathbf{1}$ is styrene and $\mathbf{2}$ is methyl methacrylate, and $r_{\mathbf{1}}$ $=0.52$ and $r_{2}=0.46 .^{22,23}$

\section{Molecular Weight and Polydispersity}

The $M_{\mathrm{n}}$ and viscometric $\left(M_{\eta}\right)$ molecular weights were measured for all samples. $M_{\mathrm{n}}$ 's for PMMA, PS, and CoSM were calculated from membrane osmometry measurements in toluene solutions at $36{ }^{\circ} \mathrm{C} . M_{\mathrm{n}}$ values for CTN were obtained by means of size exclusion chromatography of tetrahydrofuran solutions. For three of the CTN samples, this was also done from membrane osmometry in isophorone solutions, prepared by shaking them for $48 \mathrm{~h}$ at $30^{\circ} \mathrm{C}$.

$M_{\eta}$ values of all polymer samples were calculated from intrinsic viscosity through the MarkHouwink-Sakurada relationship $[\eta]=k\left(M_{\eta}\right)^{a}$, where $k$ and $a$ are system and temperature dependent quantities. The values of $k$ and $a$ used here are given in Table I.

Table II shows the characteristics of the homopolymer samples used in this work, whereas Table III lists those of the copolymers.

\section{Measurement}

Osmotic pressure measurements were done with Hewlett-Packard 501 and Knauer membrane osmometers. The regenerated cellulose membranes were provided by Sartorius and Schleicher und Schuell.

Intrinsic viscosity $[\eta]$ was determined using Schultz-Blaschke's equation ${ }^{24}$ 
Table I. Parameters of the Mark-Houwink-Sakurada Equation

\begin{tabular}{|c|c|c|c|c|}
\hline System $^{a}$ & $\begin{array}{c}T \\
\left({ }^{\circ} \mathrm{C}\right)\end{array}$ & $\begin{array}{c}10^{3} k \\
(\mathrm{~mL} / \mathrm{g})\end{array}$ & $a$ & Reference \\
\hline PMMA/chloroform & 25 & 4.85 & 0.80 & 25 \\
\hline PMMA/toluene & 30 & 7.0 & 0.73 & 22 \\
\hline PS/toluene & 30 & 12.0 & 0.71 & 22 \\
\hline $\mathrm{CoSM}_{50 / 50} /$ toluene & 30 & 8.21 & 0.74 & 28 \\
\hline CTN/acetone & 20 & 59.3 & 0.76 & 26,27 \\
\hline
\end{tabular}

${ }^{\text {a }}$ Values of $k$ and $a$ for samples of $\mathrm{CoSM}_{25 / 75}$ and $\mathrm{CoSM}_{75 / 25}$ are unknown. Hence, to characterize $\mathrm{CoSM}_{25 / 75}$, values of the PMMA/chloroform system were used, whereas in the characterization of $\mathrm{CoSM}_{75 / 25}$ we used values corresponding to $\mathrm{CoSM}_{50 / 50} /$ toluene.

$$
\frac{\eta_{\mathrm{sp}}}{c}=[\eta]+[\eta] k_{\mathrm{B}} \eta_{\mathrm{sp}}
$$

where $c$ is the solution concentration, $\eta_{\mathrm{sp}}$ is the specific viscosity, and $k_{\mathrm{B}}$ is the Schultz-Blaschke constant. $\eta_{\mathrm{sp}}$ was measured with Ostwald viscometers, where the flow time of the pure solvent was always higher than $150 \mathrm{~s}$; thus, kinetic energy corrections could be neglected. Newtonian viscosity was obtained with a Haake RV2 rotoviscometer.

\section{RESULTS}

\section{Osmotic Pressure}

Osmotic pressure as a function of the concentration was measured from the dilute to the semidi- lute regime. Some typical curves are shown in Figure 1(a). Results can be plotted as function of the overlap parameter $s$. This is made by means of eqs 2-4, adjusting $M_{\mathrm{n}}$ and $\beta$ for each sample through a least-squares fit. As shown in Figure 1(b), all data collapse nicely onto a universal curve that is well represented by the theoretical expression. The overlap parameter can be considered a universal variable.

The values $\beta$ obtained in this way are collected in the first column of Table IV for all systems. We expect from theory that $\beta$ depends on temperature and chemical composition but not on molecular weight. This independence of molecular weight seems to hold, as can be seen in Figure 2 where values of $\beta$ for $\operatorname{CoSM}_{50 / 50}$ in toluene are shown as a function of $M_{\mathrm{n}}$.

\section{Newtonian Viscosity}

Newtonian viscosity as a function of the concentration was also measured for dilute through semidilute solutions. Figure 3(a) shows some typical curves. By using eqs 2, 3, and 5-7, a universal curve can be constructed plotting $\eta_{\mathrm{sp}} /(c[\eta])$ as a function of the overlap parameter $s$, where $\beta$ is calculated for each sample by doing a leastsquares fit. Figure 3(b) indicates the experimental data as a function of the universal variable $s$ together with the theoretical curve. A good fit to the data is obtained despite the rather harsh approximations involved in eq 7 . The corresponding $\beta$ values are given in Table IV. As before, $\beta$ is independent of molecular weight, as can be seen in Figure 4 for CTN-isophorone.

Table II. Characteristics of the Homopolymer Samples Used in This Work

\begin{tabular}{|c|c|c|c|c|c|}
\hline Sample & $10^{-3} M_{\mathrm{n}}(\mathrm{g} / \mathrm{mol})$ & $\begin{array}{c}10^{-3} M_{\eta} \\
(\mathrm{g} / \mathrm{mol})\end{array}$ & $M_{\eta} / M_{\mathrm{n}}$ & Solvent & $\begin{array}{l}{[\eta]\left(\mathrm{cm}^{3} / \mathrm{g}\right)} \\
\left(\text { at } 30^{\circ} \mathrm{C}\right)\end{array}$ \\
\hline PMMA-288 & $288.7 \pm 0.1$ & $551 \pm 4$ & $1.91 \pm 0.01$ & - & - \\
\hline PMMA-336 & $336 \pm 13$ & $577 \pm 11$ & $1.72 \pm 0.10$ & Toluene & $102 \pm 1$ \\
\hline PMMA-559 & $599.7 \pm 0.6$ & $1185 \pm 8$ & $1.97 \pm 0.01$ & Chloroform & $347 \pm 2$ \\
\hline PMMA-801 & $801 \pm$ & $1615 \pm 10$ & $2.01 \pm 0.02$ & - & - \\
\hline PMMA-1201 & $1201 \pm 117$ & $1838 \pm 28$ & $1.55 \pm 0.17$ & Toluene & $246 \pm 4$ \\
\hline PS-300 & $300 \pm 4$ & $792 \pm 18$ & $2.64 \pm 0.09$ & Toluene $^{\mathrm{b}}$ & $182 \pm 3$ \\
\hline CTN-51 & $51.2 \pm$ & $98 \pm 2$ & $1.92 \pm 0.07$ & Isophorone & $280 \pm 5$ \\
\hline CTN-142 & $142.7 \pm$ & $307 \pm 8$ & $2.14 \pm 0.06$ & Isophorone & $908 \pm 29$ \\
\hline CTN-216 & $216.6 \pm$ & $420 \pm 10$ & $1.94 \pm 0.05$ & Isophorone & $1111 \pm 5$ \\
\hline CTN-277 & $277.8 \pm$ & $580 \pm 10$ & $2.09 \pm 0.04$ & Isophorone & $1479 \pm 15$ \\
\hline CTN-447 & $447 \pm 11^{\mathrm{a}}$ & $760 \pm 20$ & $1.71 \pm 0.09$ & Isophorone & $1778 \pm 30$ \\
\hline CTN-575 & $575 \pm 14^{\mathrm{a}}$ & $960 \pm 20$ & $1.67 \pm 0.07$ & Isophorone & $1839 \pm 22$ \\
\hline CTN-626 & $626 \pm 16^{a}$ & $1040 \pm 30$ & $1.67 \pm 0.09$ & Isophorone & $2197 \pm 59$ \\
\hline
\end{tabular}

${ }^{\text {a }}$ From size exclusion chromatography.

${ }^{\mathrm{b}}$ At $25^{\circ} \mathrm{C}$. 
Table III. Characteristics of the Copolymer Samples Used in This Work

\begin{tabular}{lccccc}
\hline \multicolumn{1}{c}{ Sample } & Comp. $^{\mathrm{a}}$ & $10^{-3} M_{\mathrm{n}}(\mathrm{g} / \mathrm{mol})$ & $10^{-3} M_{\eta}(\mathrm{g} / \mathrm{mol})$ & $M_{\eta} / M_{\mathrm{n}}$ & {$[\eta]\left(\mathrm{cm}^{3} / \mathrm{g}\right)^{\mathrm{c}}$} \\
\hline $\mathrm{CoSM}_{25 / 75}-1078$ & $0.33^{\mathrm{b}}$ & $1078 \pm 245$ & $2261 \pm 20$ & $2.10 \pm 0.52$ & $309 \pm 2$ \\
$\mathrm{CoSM}_{25 / 75}-2460$ & $0.33^{\mathrm{b}}$ & $2460 \pm 954$ & $3579 \pm 57$ & $1.45 \pm 0.69$ & $432 \pm 5$ \\
$\mathrm{CoSM}_{50 / 50}-227$ & $0.51^{\mathrm{b}}$ & $227 \pm 47$ & $415 \pm 9$ & $1.83 \pm 0.08$ & $118 \pm 2$ \\
$\mathrm{CoSM}_{50 / 50}-272$ & $0.51^{\mathrm{b}}$ & $272 \pm 7$ & $559 \pm 10$ & $2.06 \pm 0.09$ & $147 \pm 2$ \\
$\mathrm{CoSM}_{50 / 50}-565$ & $0.51^{\mathrm{b}}$ & $565 \pm 57$ & $1322 \pm 26$ & $2.34 \pm 0.29$ & $278 \pm 4$ \\
$\mathrm{CoSM}_{50 / 50}-759$ & 0.53 & $759 \pm 46$ & $1633 \pm 34$ & $2.16 \pm 0.18$ & $325 \pm 5$ \\
$\mathrm{CoSM}_{50 / 50}-906$ & 0.53 & $906 \pm 60$ & $1492 \pm 33$ & $1.66 \pm 0.15$ & $304 \pm 5$ \\
$\mathrm{CoSM}_{50 / 50}-945$ & 0.51 & $945 \pm 80$ & $1777 \pm 35$ & $1.88 \pm 0.20$ & $346 \pm 5$ \\
$\mathrm{CoSM}_{50 / 50}-1073$ & 0.53 & $1073 \pm 163$ & $1938 \pm 28$ & $1.81 \pm 0.31$ & $369 \pm 4$ \\
$\mathrm{CoSM}_{50 / 50}-1082$ & 0.53 & $1082 \pm 109$ & $2474 \pm 53$ & $2.29 \pm 0.28$ & $430 \pm 8$ \\
$\mathrm{CoSM}_{75 / 25}-546$ & 0.69 & $1335 \pm 19$ & $2.44 \pm 0.42$ & $280 \pm 3$ \\
$\mathrm{CoSM}_{75 / 25}-647$ & $0.69^{\mathrm{b}}$ & $546 \pm 95$ & $1599 \pm 34$ & $2.47 \pm 0.42$ & $320 \pm 5$ \\
\hline
\end{tabular}

${ }^{\text {a }}$ Styrene molar fraction.

${ }^{\mathrm{b}}$ Calculated from eq 10.

${ }^{\mathrm{c}}$ In toluene at $30{ }^{\circ} \mathrm{C}$.

Although it would be expected that the value of $\beta$ should not depend on the property being measured, we encounter different values when computing $\beta$ from osmotic pressure and viscosity. We discuss this question subsequently.

Finally, we measured $\beta$ as a function of temperature for CTN-isophorone systems. Values of $\beta$ are given in Table V. A slight decrease is observed in accordance with an exothermic behavior of the solution.

\section{Dilute Solution Viscosity}

$\beta$ can also be determined from viscosity of very dilute solutions using eq 8 . Values obtained in this way are collected in the third column of Table IV. Figure 5 depicts the fit for some representative samples. The value of $\beta$ so obtained is compatible with that for semidilute solutions.

\section{DISCUSSION AND CONCLUSIONS}

We have presented an extensive experimental study of thermodynamic and rheological properties of dilute to semidilute polymer solutions. Data for several polymer-solvent systems at different temperatures were analyzed with renormalization group theory, using de Gennes's blobs model to connect dynamic and conformational quantities. As seen in Figures 1(b), 3(b) (for the dilute-semidilute crossover), and 5 (for the infinitely dilute limit), a universal behavior is fol- lowed that is well described by theoretical predictions.

A nonuniversal quantity $\beta$ related to the microstructural characteristics of the systems was determined fitting experimental data to theoretical expressions. As predicted by theory, $\beta$ was independent of molecular weight and concentration (Figs. 2 and 4) and depended on the nature of the systems. For the sake of comparison we summarize the values of $\beta$ for each system in Table VI. One would expect (from the static RG theory at least) that $\beta$ would have the same value independent of the measured property. However, we found differences between the thermodynamic and dynamic properties. This disagreement could perhaps be ascribed to eq 7 that is derived from scaling arguments and clearly does not consider the screening of the hydrodynamic interaction at the same level as the screening of the excluded volume.

One would think of looking for an alternative RG-based scheme to describe the simultaneous screening of the excluded volume and the hydrodynamic interaction. At least one such scheme exists, that of Shiwa and coworkers. ${ }^{12-14} \mathrm{Al}-$ though it is not a unified RG description (in the sense that excluded volume is treated with $R G$, whereas the hydrodynamic interactions are considered within the mode-coupling theory), it does treat hydrodynamic screening in a theoretically more satisfying manner. However, two universal variables are still needed to describe the dynamics and statics. This scheme has the advantage 


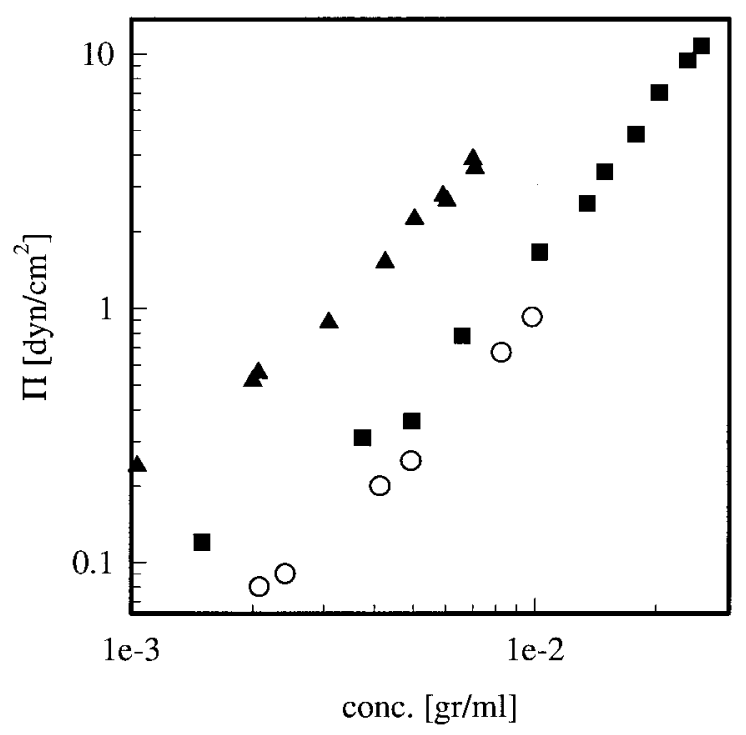

(a)

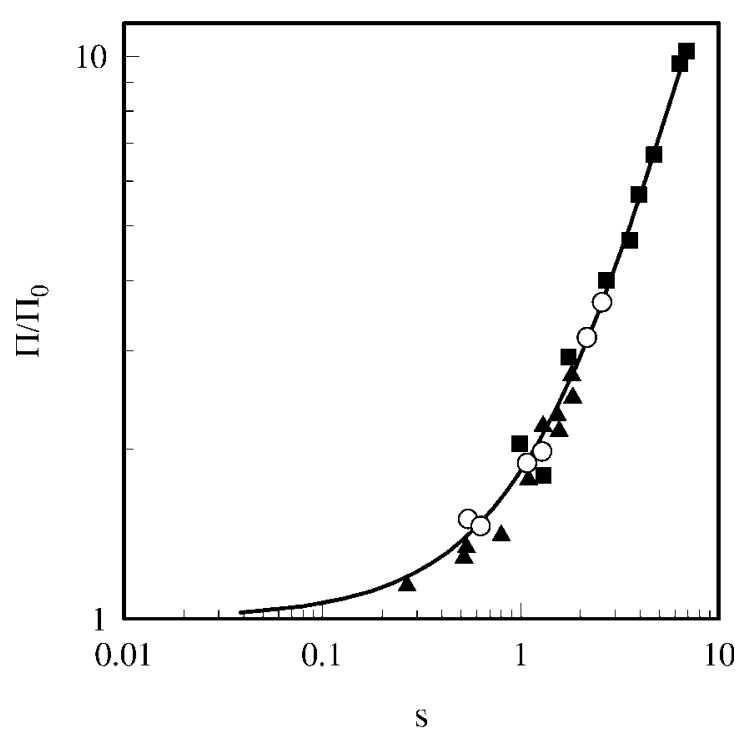

(b)

Figure 1(a). Typical data for osmotic pressure: PMMA-1201 in toluene at $36{ }^{\circ} \mathrm{C}$ (circles), $\mathrm{CoSM}_{50 / 50^{-}}$ 759 in toluene at $36{ }^{\circ} \mathrm{C}$ (squares), and CTN-142 in isophorone at $30{ }^{\circ} \mathrm{C}$ (triangles). Figure 1(b). Universal behavior of the osmotic pressure as function of the overlap parameter $s$ : PMMA-1201 in toluene at $36{ }^{\circ} \mathrm{C}$ (circles), $\mathrm{CoSM}_{50 / 50}-759$ in toluene at $36^{\circ} \mathrm{C}$ (squares), and CTN-142 in isophorone at $30{ }^{\circ} \mathrm{C}$ (triangles). The continuous curve is the theoretical prediction.

that it is based on a static, mature RG theory that can describe both temperature and concentration crossovers, which considers polydispersity and has been generalized to ternary systems. Also, on the practical side, there is (to our knowledge) no published expression for the osmotic pressure in Shiwa's scheme.

Improvements of eq 7 at this (non-RG) level are possible. Clearly, several relaxation mechanisms are found in the solution, and the viscosity is determined by the characteristic times of all of them, particularly in the crossover region. In particular, it is well known that a regime with Rouse behavior exists between the Zimm and reptation limits. One expects that explicit consideration of this regime in the model could substantially alter the dependence of Newtonian and intrinsic viscosities on concentration and molecular weight. Several models have been proposed to consider the Rouse behavior. ${ }^{2,29-32}$ All of them decouple the time dependence of the center-of-mass motion (diffusion) from stress relaxation (viscosity) and produce a pseudo 3.4 exponent. Accordingly, we also considered a model (on the basis of DoiEdwards's developments) to consider the Rouse behavior, ${ }^{2,32}$ using the blob picture as shown in the appendix to connect the parameters $a$ and $N_{\mathrm{e}}$ with $S^{2}$ and $S_{0}^{2}$ (eq A6) (see ref. 32 for details). One arrives at the following relationship between viscosity and radius of gyration (cf. with eq 7):

$$
\begin{array}{r}
\frac{\eta_{\mathrm{sp}}}{c[\eta]}=\left(\frac{S^{2}}{S_{0}^{2}}\right)^{(3 \nu-4) /(2 \nu-1)}\left\{3\left[1-\left(\frac{S^{2}}{S_{0}^{2}}\right)^{1 /(4 \nu-2)}\right]^{3}\right. \\
\left.+\left(\frac{S^{2}}{S_{0}^{2}}\right)^{3 /(4 v-2)}\right\}
\end{array}
$$

This equation correctly predicts a power law relationship between viscosity and molecular weight with a 3.4 exponent. The theoretical prediction of eq 12 is represented as a dotted line in Figure 3(b). Within the experimental range (which covers essentially the transition between dilute and semidilute regimes), a fit with this model produces values of $\beta 5-15 \%$ higher than those found with eq 7 . In the dilute limit, one finds from eq 12

$$
\frac{\eta_{\mathrm{sp}}}{c} \approx[\eta]-[\eta](6 \nu-5)(4 / 5)^{3 v} \beta^{3} M_{\mathrm{n}}^{3 \nu-1} c
$$

and with this equation we obtain values of $\beta 45 \%$ greater than those found with eq 9 (the values obtained with eqs 12 and 13 remain compatible with each other within experimental uncertainties). Therefore, even if explicit inclusion of the Rouse regime seems to improve the situation (in the sense that $\beta$ 's obtained from different proper- 
Table IV. Values of $\beta$ for All Systems Used in This Work

\begin{tabular}{|c|c|c|c|c|}
\hline Polymer & Solvent & $\beta$ (Osmosis) & 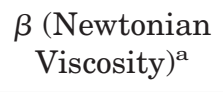 & $\begin{array}{c}\beta \text { (Dilute Solution }^{\text {Viscosity) }} \\
\text { Vis }^{\mathrm{a}}\end{array}$ \\
\hline PS-300 & Toluene & $0.2137(2)^{\mathrm{b}}$ & - & - \\
\hline $\mathrm{CoSM}_{75 / 25^{-}}-546$ & Toluene & $0.22 \quad(2)^{\mathrm{b}}$ & $0.117(5)$ & - \\
\hline $\mathrm{CoSM}_{75 / 25^{-647}}$ & Toluene & $0.215(5)^{\mathrm{b}}$ & $0.120(4)$ & - \\
\hline $\mathrm{CoSM}_{50 / 50}-227$ & Toluene & $0.205(3)^{\mathrm{b}}$ & - & $0.118(4)$ \\
\hline $\mathrm{CoSM}_{50 / 50}-272$ & Toluene & $0.1992(9)^{b}$ & $0.117(1)$ & $0.112(5)$ \\
\hline $\mathrm{CoSM}_{50 / 50^{-}}-565$ & Toluene & $0.204(4)^{\mathrm{b}}$ & - & $0.108(5)$ \\
\hline $\mathrm{CoSM}_{50 / 50^{-759}}$ & Toluene & $0.2043(7)^{\mathrm{b}}$ & $0.116(2)$ & $0.107(5)$ \\
\hline $\mathrm{CoSM}_{50 / 50}-906$ & Toluene & $0.204(1)^{\mathrm{b}}$ & - & $0.100(8)$ \\
\hline $\mathrm{CoSM}_{50 / 50^{-}}-945$ & Toluene & $0.1890(8)^{b}$ & $0.121(3)$ & $0.106(5)$ \\
\hline $\mathrm{CoSM}_{50 / 50}-1073$ & Toluene & $0.199(2)^{\mathrm{b}}$ & 0.118 & $0.110(2)$ \\
\hline $\operatorname{CoSM}_{50 / 50}-1082$ & Toluene & $0.202(7)^{\mathrm{b}}$ & 0.120 & 0.096 \\
\hline $\mathrm{CoSM}_{25 / 75}-1078$ & Toluene & $0.20 \quad(3)^{\mathbf{b}}$ & $0.106(6)$ & - \\
\hline $\mathrm{CoSM}_{25 / 75}-2460$ & Toluene & $0.204(5)^{b}$ & $0.10 \quad(1)$ & - \\
\hline PMMA-336 & Toluene & $0.183(4)^{\mathrm{b}}$ & - & - \\
\hline PMMA-559 & Toluene & $0.165(3)^{\mathrm{b}}$ & - & - \\
\hline PMMA-801 & Toluene & $0.188 \quad(2)^{\mathrm{b}}$ & - & - \\
\hline PMMA-1201 & Toluene & $0.181(4)^{b}$ & - & - \\
\hline PMMA-288 & Isophorone & $0.169(4)^{\mathrm{a}}$ & - & - \\
\hline $\mathrm{CoSM}_{25 / 75}-1078$ & Isophorone & - & $0.116(4)$ & 0.09 \\
\hline $\mathrm{CoSM}_{25 / 75}-2460$ & Isophorone & - & $0.103(5)$ & $0.075(5)$ \\
\hline CTN-51 & Isophorone & $0.275(2)^{\mathrm{a}}$ & - & $0.200(2)$ \\
\hline CTN-142 & Isophorone & $0.31 \quad(1)^{\mathrm{a}}$ & $0.271(5)$ & $0.255(9)$ \\
\hline CTN-216 & Isophorone & $0.30 \quad(1)^{\mathrm{a}}$ & $0.269(2)$ & 0.205 (1) \\
\hline CTN-277 & Isophorone & $0.350(7)^{\mathrm{a}}$ & $0.256(1)$ & $0.244(1)$ \\
\hline CTN-447 & Isophorone & - & $0.274(6)$ & 0.255 \\
\hline CTN-575 & Isophorone & - & $0.272(4)$ & $0.200(2)$ \\
\hline CTN-626 & Isophorone & - & $0.297(3)$ & $0.221(3)$ \\
\hline
\end{tabular}

Errors are given in parenthesis (precision in the last decimal place).

${ }^{\text {a }} T=30^{\circ} \mathrm{C}$.

${ }^{\mathrm{b}} \mathrm{T}=36^{\circ} \mathrm{C}$.

ties are closer to each other), it is not enough to explain the observed differences, at least at this non-RG level and without introducing additional adjustable constants.

To avoid introducing new fitting parameters, we can stick to eq 7 , which (although admittedly crude in its approximations) gives a very simple connection with the static RG theory. Then, accepting that a different constant is needed for dynamic properties, a reasonable quantitative agreement can be achieved within a unified scheme. These results together with our previous study $^{11}$ of relaxation times seem to indicate that one $\beta$ is enough to describe all dynamic properties. In light of these results, we suggest that by measuring two quantities $\beta_{\text {therm }}$ and $\beta_{\text {dyn }}$, the behavior of polymer solutions across the whole dilute-semidilute crossover can be predicted in a unified framework. This could considerably sim-

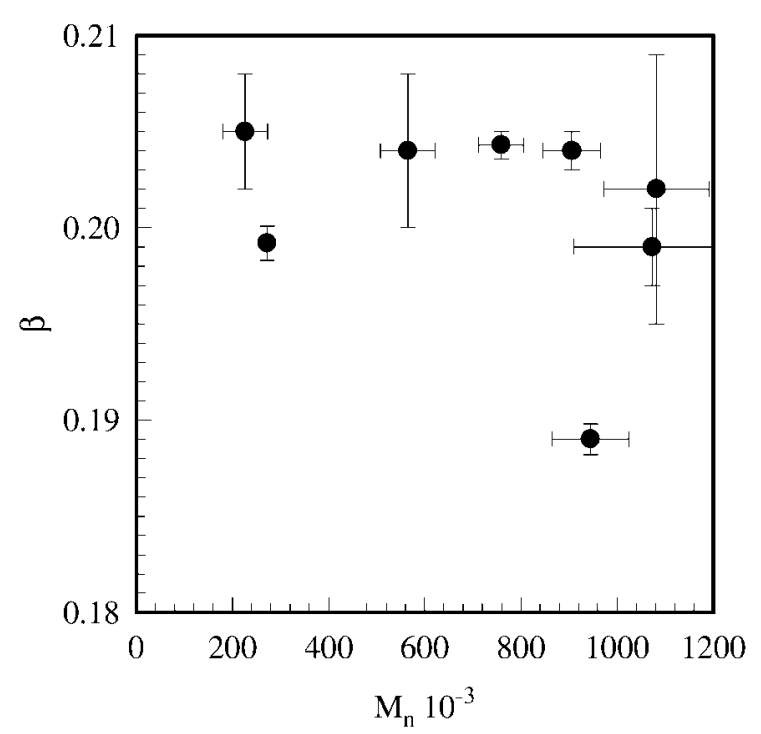

Figure 2. Dependence of $\beta$ values, as determined from osmotic pressure data, on molecular weight. System: $\mathrm{CoSM}_{50 / 50}$ in toluene at $36{ }^{\circ} \mathrm{C}$. 


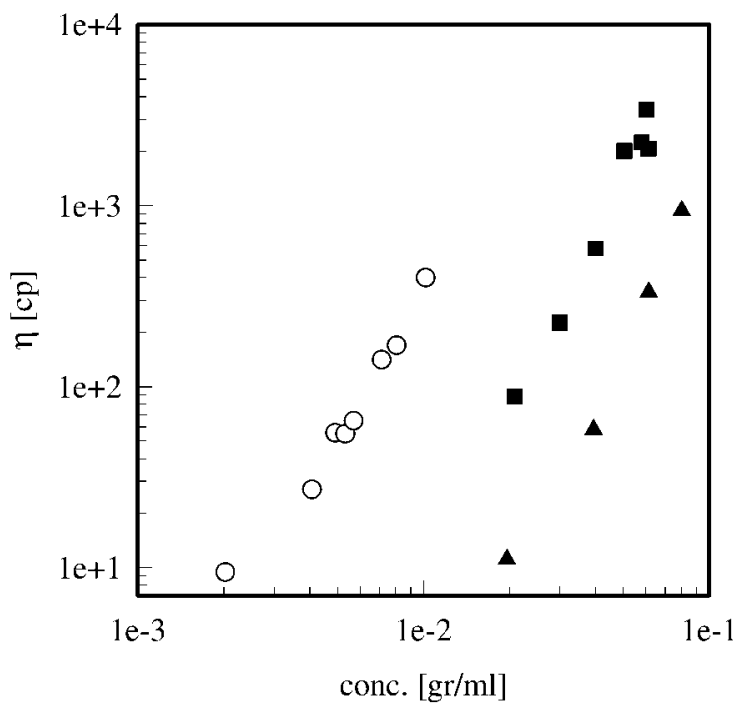

(a)

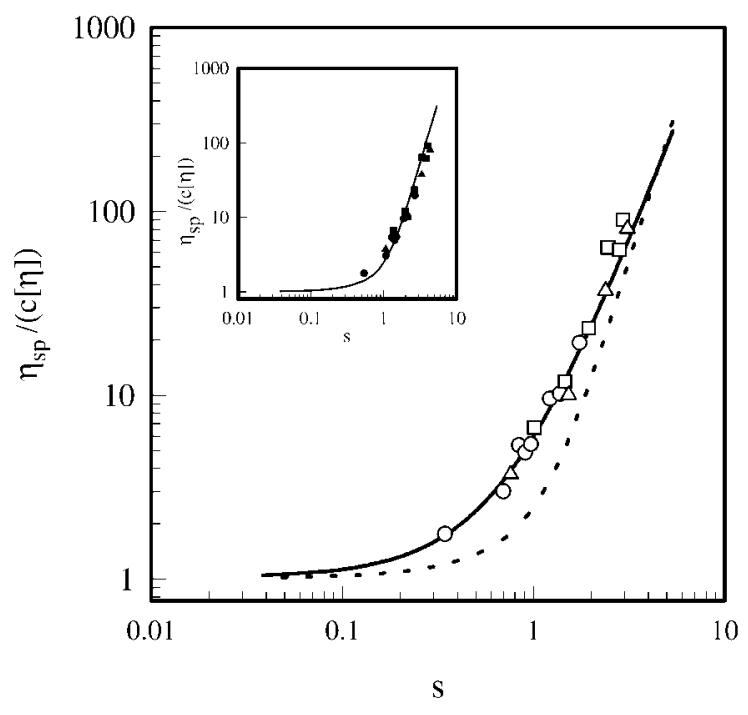

(b)

Figure 3(a). Typical data for Newtonian viscosity at $T=30^{\circ} \mathrm{C}$ : CTN-142 in isophorone (circles), $\mathrm{CoSM}_{50 / 50^{-}}$ 759 in toluene (squares), and $\mathrm{CoSM}_{75 / 25}-546$ in toluene (triangles). Figure 3(b). Universal behavior of viscosity as function of the overlap parameter $s$ at $T=30{ }^{\circ} \mathrm{C}$ : $\mathrm{CTN}-142$ in isophorone (circles), $\mathrm{CoSM}_{50 / 50^{-759}}$ in toluene (squares), and $\mathrm{CoSM}_{75 / 25}-546$ in toluene (triangles). The continuous curve is the theoretical prediction according to eq 7 , and the dotted line is the prediction according to eq 12. The main figure shows the fit to eq 7 , whereas the inset is the fit to eq 12 .

plify the experimental characterization of polymer solutions, important for laboratory as well as industrial applications.

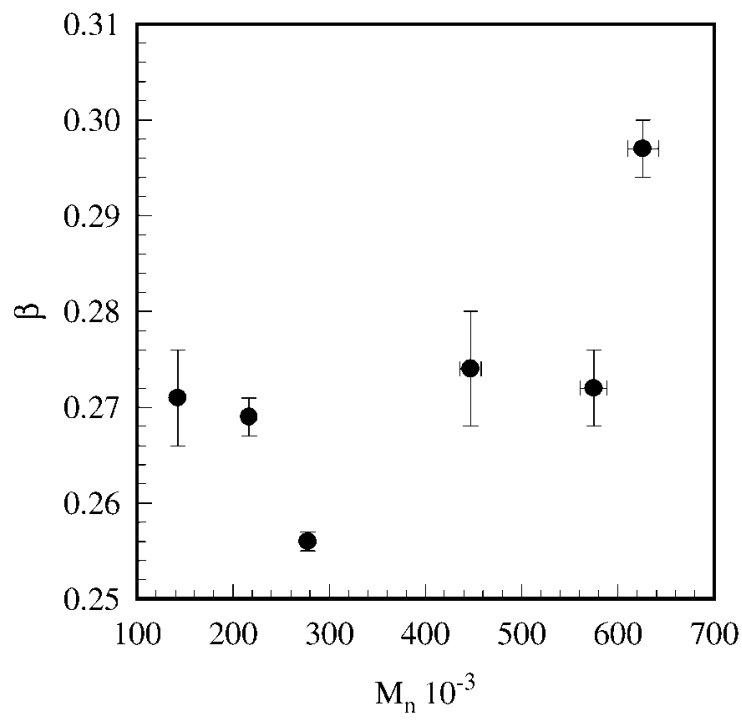

Figure 4. Dependence of $\beta$ values, as determined from viscosity data, on molecular weight. System: CTN in isophorone at $30^{\circ} \mathrm{C}$.

The authors thank F. Amarilla, A. Pesce, and M. A. Sanservino for the very competent technical assistance. This work was funded by Consejo Nacional de Investigaciones Científicas y Técnicas (CONICET, Argentina), Comisión de Investigaciones Científicas de la Provincia de Buenos Aires (CIC, Argentina), and Universidad Nacional de La Plata. R. V. Figini and M. Marx-Figini are members of the Carrera del Investigador of CONICET, and M. S. Cortizo is a member of the Carrera del Profesional de Apoyo of the same institution. I. M. Irurzun and T. S. Grigera are research fellows of CONICET.

\section{APPENDIX}

Equation 7 is obtained as follows. ${ }^{11}$ According to the reptation model, a chain occupies a tubelike

Table V. Values of $\beta$ as a Function of Temperature, from Newtonian Viscosity, for Cellulose TrinitrateIsophorone

\begin{tabular}{lcccc}
\hline Sample & $\begin{array}{c}T=20 \\
{ }^{\circ} \mathrm{C}\end{array}$ & $\begin{array}{c}T=30 \\
{ }^{\circ} \mathrm{C}\end{array}$ & $\begin{array}{c}T=50 \\
{ }^{\circ} \mathrm{C}\end{array}$ & $\begin{array}{c}T=70 \\
{ }^{\circ} \mathrm{C}\end{array}$ \\
\hline CTN-51 & $0.226(3)$ & - & - & - \\
CTN-142 & $0.278(2)$ & $0.271(5)$ & $0.261(3)$ & $0.249(6)$ \\
CTN-216 & $0.279(2)$ & $0.269(2)$ & $0.259(4)$ & $0.246(4)$ \\
CTN-277 & $0.263(2)$ & $0.256(1)$ & $0.238(2)$ & $0.220(5)$ \\
CTN-447 & $0.285(4)$ & $0.274(6)$ & $0.248(8)$ & $0.241(6)$ \\
CTN-575 & $0.300(6)$ & $0.272(4)$ & $0.264(8)$ & $0.256(9)$ \\
CTN-626 & - & $0.297(3)$ & $0.284(2)$ & $0.280(3)$ \\
\hline
\end{tabular}

Errors are given in parenthesis (precision in the last decimal place). 


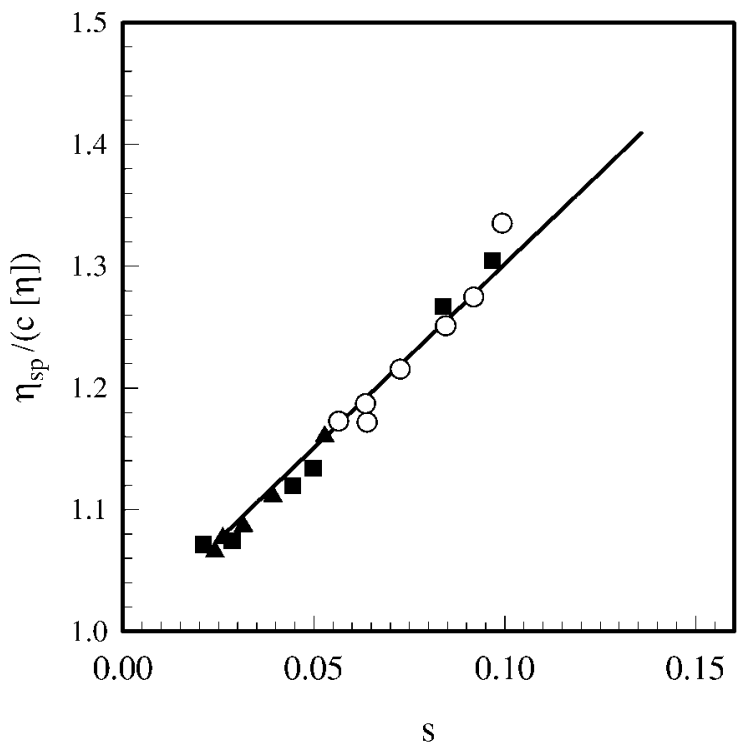

Figure 5. Universal behavior in the dilute limit at $T$ $=30{ }^{\circ} \mathrm{C}$ : CTN-142 in isophorone (circles), $\mathrm{CoSM}_{25 / 75^{-}}$ 2460 in isophorone (squares), and CTN-447 in isophorone (triangles). The continuous curve is the theoretical prediction, eq 8 .

region, which it vacates as it diffuses, creating a new tube around itself. The dynamics of this process were discussed by de Gennes ${ }^{33}$ to determine the low-frequency properties of entangled polymers using two concentration-dependent parameters $a$ and $N_{\mathrm{e}}$-the distance and the number of monomers between entanglements. Consider a chain that consists of a series of segments of size proportional to $a$. The total number of segments is proportional to $N / N_{\mathrm{e}}$, and (assuming the hydrodynamic interaction is not screened within the segments) the chain-friction coefficient $f_{\text {chain }}$ will be (using the Stokes-Einstein relation)

$$
f_{\text {chain }} \propto 6 \pi \eta_{\mathrm{s}} a\left(\frac{N}{N_{\mathrm{e}}}\right) \propto \frac{k T}{D_{\text {tube }}}
$$

where $N$ is the number of monomers, and $\eta_{\mathrm{s}}$ is the solvent viscosity. To disengage from a given tube, the molecule must diffuse a distance of the order of the chain length $a N / N_{\mathrm{e}}$. Therefore, the longest relaxation time $\tau_{\mathrm{r}}$ will be

$$
\tau_{\mathrm{r}} \propto \frac{1}{2} \frac{\left(a \frac{N}{N_{\mathrm{e}}}\right)^{2}}{D_{\text {tube }}}
$$

The polymer contribution to the solution viscosity, $\eta-\eta_{\mathrm{s}}$ is obtained by assuming a Maxwell fluid

$$
\eta-\eta_{\mathrm{s}} \propto \tau_{\mathrm{r}} G
$$

where $G$ is the relaxation strength associated with $\tau_{\mathrm{r}}$, and $\eta$ is the solution viscosity. Assuming that the instantaneous network of entanglements behaves like a rubber, the modulus $G$ is proportional to the number concentration of entanglements $^{34}$

$$
G \propto \frac{c}{N_{\mathrm{e}}}
$$

where $c$ is the weight polymer concentration. From eqs A1-A4 it is possible to obtain the reduced viscosity

\begin{tabular}{|c|c|c|c|c|c|}
\hline Polymer & Solvent & $T\left({ }^{\circ} \mathrm{C}\right)$ & $\beta$ (Osmosis) & $\begin{array}{l}\beta \text { (Newtonian } \\
\text { Viscosity) }\end{array}$ & $\begin{array}{c}\beta \text { (Dilute Solution } \\
\text { Viscosity) }\end{array}$ \\
\hline PS & Toluene & 36 & $0.2137(2)$ & - & - \\
\hline $\mathrm{CoSM}_{75 / 25}$ & Toluene & 36 & $0.22 \quad$ (1) & $0.118(6)^{\mathrm{a}}$ & - \\
\hline $\mathrm{CoSM}_{50 / 50}$ & Toluene & 36 & $0.200 \quad(6)$ & $0.118(5)^{\mathrm{a}}$ & $0.11(1)^{\mathrm{a}}$ \\
\hline $\mathrm{CoSM}_{25 / 75}$ & Toluene & 36 & 0.20 & $0.10 \quad$ (1) & - \\
\hline PMMA & Toluene & 36 & $0.18 \quad$ (1) & - & - \\
\hline PMMA & Isophorone & 30 & 0.169 & - & - \\
\hline $\mathrm{CoSM}_{25 / 75}$ & Isophorone & 30 & - & $0.11 \quad$ (1) & $0.08 \quad(2)$ \\
\hline CTN & Isophorone & 30 & $0.31 \quad$ (3) & $0.27 \quad(2)$ & 0.22 \\
\hline
\end{tabular}

$$
\frac{\eta_{\mathrm{sp}}}{c} \propto \frac{\left(\frac{N a}{N_{\mathrm{e}}}\right)^{3}}{N_{\mathrm{e}}}
$$

Table VI. Summary of $\beta$ for Each System

$$
{ }^{\mathrm{a}} \mathrm{T}=30{ }^{\circ} \mathrm{C} \text {. }
$$


To arrive at an expression in terms of molecular parameters, we argue that $a$ is proportional to the correlation length, identifying the segments of the entanglement picture with the blobs of the static description of semidilute solutions. ${ }^{35}$ Thus, the scaling relations of the blob picture allow us to write

$$
a \propto N_{\mathrm{e}}^{\nu} ; \quad S_{0} \propto N^{\nu} ; \quad S \propto a\left(\frac{N}{N_{\mathrm{e}}}\right)^{1 / 2}
$$

From eqs A5 and A6 we then obtain

$$
\frac{\eta_{\mathrm{sp}}}{c} \propto \frac{S_{0}^{3}}{N}\left(\frac{S^{2}}{S_{0}^{2}}\right)^{(3 \nu-4) /(2 \nu-1)}
$$

Although obtained for the semidilute solution, eq A7 has the correct dilute solution behavior, namely, Zimm's result

$$
[\eta] \propto \frac{S_{0}^{3}}{N}
$$

Then eqs A7 and A8 lead to eq 7, where we use an equals sign because the proportionality constant must be 1 , as seen from the infinite dilution limit.

Equation 7 yields de Genne's relation in the semidilute limit $(w \rightarrow 0 ; \mathrm{s} \rightarrow \infty)$; then

$$
\eta_{\mathrm{sp}} /(c[\eta]) \propto w^{(3 v-4)} \propto s^{((3 v-4) /(1-3 v))}
$$

and so

$$
\eta_{\mathrm{sp}} \rightarrow M^{3} c^{3 /(3 \nu-1)} \quad(w \rightarrow 0)
$$

We use this equation across the whole crossover, although clearly outside the range of validity of the arguments used to obtain it. As shown, the dilute and semidilute limits are described correctly. In the crossover region, the renormalization group (RG) expression has the effect of interpolating smoothly between the limits.

Finally, to obtain the dilute limit $(w \rightarrow 1)$ of eq 7 , we begin expanding eq 2 up to first order in (1 $-w)$ to get

$$
s=\frac{1}{2}\left(\frac{5}{4}\right)^{3 v}(1-w)+O(1-w)^{2}
$$

Then the radius of gyration (eq 5) is written at first order as

$$
\frac{S^{2}}{S_{0}^{2}}=1+(2 \nu-1)(w-1)+O(w-1)^{2}
$$

where we have neglected the influence of polydispersity, $[1+\Delta(w)] /[1+\Delta(1)] \approx 1$. Equations A11, $\mathrm{A} 12$, and 7 then yield eq 8 .

\section{REFERENCES AND NOTES}

1. Yamakawa, H. Modern Theory of Polymer Solutions; Harper \& Row: New York, 1971.

2. Doi, M.; Edwards, S. F. The Theory of Polymer Dynamics; Oxford University Press: Oxford, 1986.

3. de Gennes, P. G. Scaling Concepts in Polymer Physics; Cornell University Press: Ithaca, NY, 1979.

4. des Cloizeaux, J.; Jannink, G. Les polymères en solution: leur modélisation et leur structure, Editions de Physique: Les Ulis; 1987.

5. des Cloizeaux, J. J Phys (Paris) 1975, 36, 281.

6. Oono, Y.; Kohomoto, M. J Chem Phys 1983, 78(1), 1.

7. Oono, Y.; Ohta, T.; Freed, K. F. J Chem Phys 1981, 74,6458 .

8. Schäfer, L. Macromolecules 1982, 15, 652.

9. Schäfer, L. Macromolecules 1984, 17, 1357.

10. Schäfer, L. Excluded Volume Effects in Polymer Solutions; Springer: Berlin, 1999.

11. Irurzun, I. M.; Grigera, J. R.; Figini, R. V.; MarxFigini, M. Polymer 2000, 41, 725.

12. Shiwa, Y. Phys Rev Lett 1986, 58, 2102.

13. Shiwa, Y.; Oono, Y.; Baldwin, P. R. Macromolecules 1988, 21, 208.

14. Nyström, B. Macromolecules 1993, 26, 3784 and references therein.

15. Irurzun, I. M.; Grigera, T. S.; Cortizo, M. S.; Figini, R. V.; Marx-Figini, M. to be published, 2001. For the development of L. Schäfer's theory to ternary solutions see Kappeler, C.; Schäfer, L. Macromolecules 1990, 23, 2766; Schäfer, L.; Kappeler, C. Colloid Polym Sci 1990, 268, 995; Schäfer, L.; Lehr, U.; Kappeler, C. J Phys (Paris) 1991, 1, 211; and Schäfer, L.; Kappeler, C. J Chem Phys 1993, 99, 8.

16. Noda, I.; Kato, N.; Kitano, T.; Nagasawa, M. Macromolecules 1981, 14, 668.

17. Takahashi, Y.; Isono, Y.; Noda, I.; Nagasawa, M. Macromolecules 1985, 18, 1002.

18. $\mu$ corresponds to the quantity $\beta$ of ref. 9. We have changed the symbol to avoid confusion with eq 3 .

19. This expression of $\Delta(w)$ was obtained from Figure 6 of ref. 9 fitting a polynomial function.

20. Marx-Figini, M. Makromol Chem 1961, 50, 196.

21. Alfrey, T., Jr.; Bohrer, J. J.; Mark, H. Copolymerization; Interscience: New York, 1952.

22. Young, L. J. In Polymer Handbook, 2nd ed.; Brandup, J.; Immergut, E. H.; McDowell, W., Eds.; Wiley: New York, 1975. 
23. Lewis, F. M.; Walling, C.; Cummings, W.; Briggs, E. R.; Mayo, F. R. J Am Chem Soc 1948, 70, 1519.

24. Polymer Molecular Weights, Part II; Slade, P. E., Jr., Ed.; Marcel Dekker: New York, 1975.

25. Wagner, H. L. J Phys Chem Ref Data 1987, 16, 165.

26. Marx, M.; Schulz, G. V. Makromol Chem 1959, 31, 140.

27. Marx-Figini, M.; Schulz, G. V. Makromol Chem 1962, 54, 102.

28. Tanaka, T.; Kotaka, T.; Bau, K.; Hattori, M.; Inagaki, H. Macromolecules 1977, 10, 960.

29. Lin, Y. H. Macromolecules 1984, 17, 2846.

30. Lin, Y. H. Macromolecules 1989, 22, 1437.
31. Wool, R. P. Macromolecules 1993, 26, 7.

32. Irurzun, I. M. Ph.D. Thesis, University of La Plata, Argentina, 1998.

33. de Gennes, P.-G. J Chem Phys 1971, 55, 572.

34. Treolar, L. R. G. The Physics of Rubber Elasticity; Oxford University Press: London, 1975.

35. Actually the number of entanglements in a chain is less than the number of blobs; therefore, the number of blobs per entanglement $N_{\mathrm{e}}^{*}$ should be introduced. We choose to avoid this new unknown constant that does not change the semidilute scaling of eq A7. 